Research Article

\title{
Convergence in Bucket Brigades in a Tree-Shaped Picking System
}

\author{
Xiangbin $\mathrm{Xu},{ }^{1,2}$ Caige $\mathrm{Xu},{ }^{2}$ and Feng Shi ${ }^{1}$ \\ ${ }^{1}$ School of Traffic and Transportation Engineering, Central South University, Changsha, Hunan 410075, China \\ ${ }^{2}$ School of Mechanical and Electrical Engineering, East China Jiaotong University, Nanchang, Jiangxi 330013, China \\ Correspondence should be addressed to Feng Shi; shifeng@csu.edu.cn
}

Received 25 February 2014; Revised 16 May 2014; Accepted 21 May 2014; Published 22 June 2014

Academic Editor: Gualberto Solís-Perales

Copyright (c) 2014 Xiangbin Xu et al. This is an open access article distributed under the Creative Commons Attribution License, which permits unrestricted use, distribution, and reproduction in any medium, provided the original work is properly cited.

\begin{abstract}
Previous works on the dynamics of bucket brigades have mainly focused on linear production systems or tree-shaped assembly systems with insignificant walk-back time, but this assumption is invalid for most city picking systems. We propose a two-truck bucket brigade tree-shaped picking system when truck walk-back time is significant and analyze the dynamics of the system in each region of the parameter space, showing that relatively complex picking systems can enjoy the benefit of self-balance with minimal managerial intervention and provide insights and operating principles for the implementation and management of the bucket brigade picking system.
\end{abstract}

\section{Introduction}

The term "bucket brigade" was coined by Bartholdi III and Eisenstein [1] for the TSS line [2]. They provided the first comprehensive analysis of the dynamics of such systems and pointed out that if workers can be sequenced from the slowest to the fastest along the production line, then there is a stable fixed point at which the system will converge, independent of the initial positions of the workers. Furthermore, bucket brigade systems yield optimal throughput. Since then, the bucket brigade has been intensively studied with the consideration of stochastic processing time [3], high labor turnover [4], significant walk-back time [5], undominated workers' speeds [6], workers' learning ability [7], and so forth. As a highly effective and self-balanced system, the bucket brigade has also been widely used in many real-life systems such as production lines and order picking systems in distribution centers $[1,4,8-10]$.

So far, researches on bucket brigade systems have mainly focused on continuously linear or U-shaped [11] production or picking lines. Only Lim [12] and Bartholdi III et al. [13] introduced the bucket brigade to complex assembly lines, describing the assembly of products with two subcomponents as a tree-shaped assembly line, but their models did not take the worker's walk-back time into consideration because it can be neglected since workers assemble products in just one location, which may make sense when they assemble products on the same factory floor. There are, however, several real-life systems in which worker's walk-back time should not be neglected, and the city picking system is a typical example. Therefore, walk-back time might be significant when we introduce the bucket brigade into the city picking system because of the long distance of the picking route.

In this paper, we propose a bucket brigade tree-shaped city picking system in which only two trucks pick items in tree-shaped picking routes; trucks walk back, not instantaneously but at a constant velocity. We analyze the dynamics of such a system and find that self-organization can be realized in different situations. To the best of our knowledge, this represents the first analysis of bucket brigades in such an environment. Although the model we present in this paper is simple, our results offer a basis for studying complex systems with more trucks and complicated picking routes, even networks. Given the widespread presence of assembly and logistics networks, our work offers a very constructive guide for implementing tree-shaped, even network-based, bucket brigade systems. The paper is organized as follows. In Section 2, we propose a two-truck bucket brigade tree-shaped 


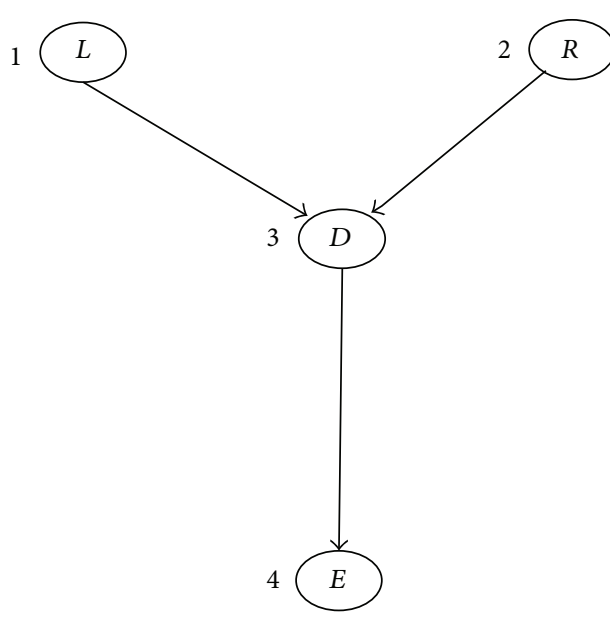

(a) Structure of picking system

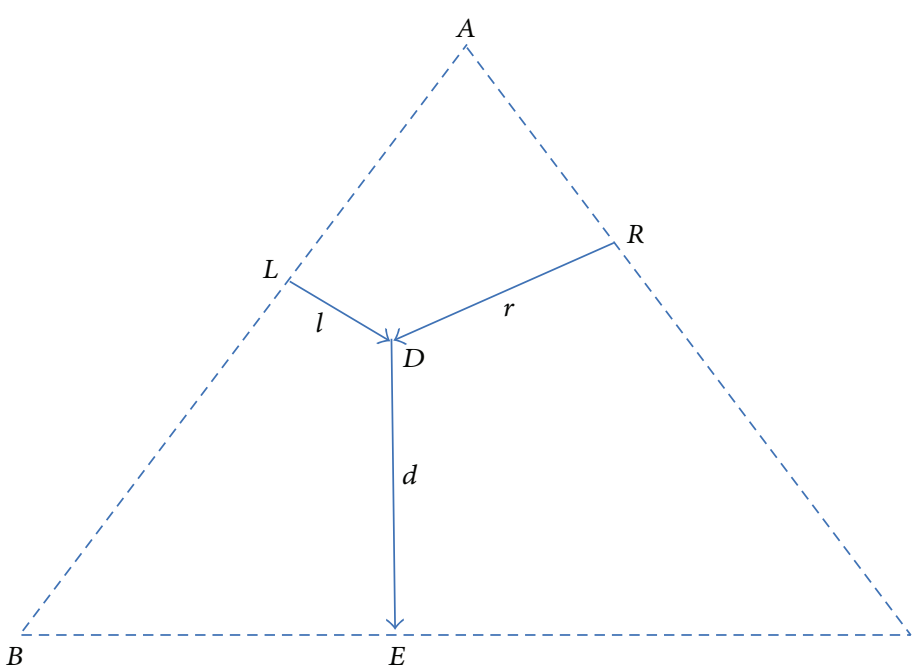

(b) Picking system embedded in an equilateral triangle

FIGURE 1: A two-truck bucket brigade tree-shaped picking system.

picking system. In Section 3, we analyze the convergence of the system. In Section 4, we present the system's convergence and stability simulations. Section 5 concludes.

\section{Model}

Because of the complexity of the analysis, we consider a simple two-truck bucket brigade tree-shaped picking system, and the tree is a simple "Y" shape as shown in Figure 1(a). The tree consists of only three arcs, each of which corresponds to a subpicking route. Subpicking routes $L \rightarrow D$ and $R \rightarrow D$ are initiated at nodes $L$ and $R$, respectively, and are joined at node $D$ to the depot $E$ by the subpicking route $D \rightarrow E$. The items to be picked are uniformly distributed over subpicking routes $L \rightarrow D, R \rightarrow D$, and $D \rightarrow E$, respectively; all items must be picked and sent to depot $E$ by two trucks. Each new picking task starts at the initial node $(L$ or $R)$ and is completed at node $E$. Under the operation of a two-truck bucket brigade, both trucks are sequenced from slower to faster at the same initial node $(L$ or $R$ ) and start their picking task at their own working velocity along the subpicking route according to the bucket brigade rules. For simplicity, we denote the subpicking route by the start node; that is, $L, R$, and $D$ represent subpicking routes $L \rightarrow D, R \rightarrow D$, and $D \rightarrow E$, respectively.

\subsection{Assumption}

Assumption 1. The picking task at each subpicking route is deterministic and is spread continuously and uniformly over the corresponding subpicking route.

Thus, the picking task on subpicking routes $L, R$, and $D$ can be represented by their length $l, r$, and $d$, respectively. To simplify our analysis, we assume that the entire structure of the picking routes is embedded in an equilateral triangle $A B C$ as described in Figure 1(b). Subpicking routes $L, R$, and $D$ are perpendicular to the edges $\mathrm{AB}, \mathrm{AC}$, and $\mathrm{BC}$, respectively. It can be shown easily that the sum of the lengths $l, r$, and $d$ is a constant for the position of node $D$ arbitrarily located within the triangle. For simplicity, we normalize the total picking task on the system to one, so that $l+r+d=1$.

Assumption 2. Each truck $i=1,2$ is characterized by a work velocity $v_{i}$ and both trucks walk back with the same velocity $v_{r} ; v_{1}, v_{2}$, and $v_{r}$ are fixed and constant over all subpicking routes and satisfy $v_{1}<v_{2}<v_{r}$.

Assumption 3. Trucks must travel along the picking routes and they are not allowed to pass each other.

Assumption 4. The trucks' unproductive walk-back time cannot be neglected.

Assumption 5. The handover time is negligible compared with the picking time.

2.2. Rules. Let both trucks be numbered from slower to faster 1 and 2. Truck 1 is the predecessor of truck 2, and truck 2 is the successor of truck 1 . Since the picking routes in our model are tree-shaped, the events in our model are a little different from previous linear bucket brigade models. In order to adapt the tree-shaped picking system to a linear bucket brigade system, we need to serialize the tree-shaped picking routes into a sequence of subpicking routes. According to Lim [12], the tree-shaped picking routes in our model are serialized in two ways: $L \rightarrow R \rightarrow D$ or $R \rightarrow L \rightarrow D$. After serialization, both trucks work in bucket brigade way according to the following rules.

Forward. Pick your item moving from one subpicking route to the next according to the serialized ordering. If you are the last truck and complete a picking task or if your picked items are taken over by your successor, then go Back. 
Back. If you are the first truck, then return to the first subpicking route according to the serialized ordering and start a new picking task and go Forward; otherwise, walk back to your predecessor, take over its picked items, and go Forward.

\section{Convergence Analysis}

Just like Lim [12], here, we also consider the case in which node $D$ is in the left part of the triangle shown in Figure 1(b); that is, $l \leq r$. The analysis for the case $l>r$ is similar. We define the parameter:

$$
s \equiv \frac{1 / v_{2}+1 / v_{r}}{1 / v_{1}+1 / v_{2}+2 / v_{r}}
$$

to present the relative working time of the trucks, $0<s<$ $1 / 2$. The tree-shaped picking system can be serialized into different sequences depending on the values of $s, l$, and $r$. Define the following four disjoint regions.

Region 1. $s<l$.

Region 2. $l<s<r$.

Region 3. $r<s<l+r$.

Region 4. $s>l+r$.

According to Lim [12], each region has different ways of serializing the picking routes to make the two-truck bucket brigade tree-shaped picking system balance.

Let $x^{k}$ represent the position of the $k$ th hand-off on the tree-shaped picking system in Figure 1(b); when $x^{k} \in[0, l]$, $x^{k} \in[l, l+r]$, and $x^{k} \in[l+r, 1]$, the hand-off position is located on routes $L, R$, and $D$, respectively. At each iteration $k$, truck 1 hands items collected in $k$ period to truck 2 at position $x^{k}$; then truck 1 returns to the first subpicking route (according to the sequenced picking route) with velocity $v_{r}$ to begin its $k+1$ period picking task, while truck 2 continues collecting items on its $k$ period picking task. When truck 2 completes its $k$ period picking task, it goes back with velocity $v_{r}$ to receive items collected in $k+1$ period from truck 1 at $x^{k+1}$. Different from Lim [12], the trucks' walk-back time is not insignificant and the synchronization between truck 1 and truck 2 in subpicking routes is not a must here.

3.1. Region $1(s<l)$. In Region 1 , there are two different ways to serialize the tree-shaped picking system to make the twotruck bucket brigade picking system balance. Lemma 6 shows the existence and uniqueness of a fixed point on the treeshaped picking system under sequence $L \rightarrow R \rightarrow D$.

Lemma 6. If the tree-shaped picking system is sequenced as $L \rightarrow R \rightarrow D$ in Region 1, then there exists a unique fixed point on route $L$ and it is given by

$$
x_{L}^{*}=\frac{1 / v_{2}+1 / v_{r}}{1 / v_{1}+1 / v_{2}+2 / v_{r}} \text {. }
$$

Proof. If the order of picking routes is sequenced as $L \rightarrow$ $R \rightarrow D$, the fixed point $x_{L}^{*}$ can be found by solving

$$
\frac{x_{L}^{*}}{v_{r}}+\frac{x_{L}^{*}}{v_{1}}=\frac{1-x_{L}^{*}}{v_{2}}+\frac{1-x_{L}^{*}}{v_{r}},
$$

$x_{L}^{*}=s<l$, so the fixed point is located on route $L$.

Lemma 7. If the tree-shaped picking system is sequenced as $L \rightarrow R \rightarrow D$ in Region 1 and

$$
x^{0} \in\left[\max \left(0,1-\frac{1 / v_{1}+1 / v_{r}}{1 / v_{2}+1 / v_{r}} l\right), l\right],
$$

then

$$
\begin{array}{r}
x^{k} \in\left[\max \left(0,1-\frac{1 / v_{1}+1 / v_{r}}{1 / v_{2}+1 / v_{r}}\right), l\right], \\
\text { for } k=1,2,3 \ldots
\end{array}
$$

Proof. We first prove that if

$$
x^{k-1} \in\left[\max \left(0,1-\frac{1 / v_{1}+1 / v_{r}}{1 / v_{2}+1 / v_{r}} l\right), l\right] \text {, }
$$

then

$$
x^{k} \in\left[\max \left(0,1-\frac{1 / v_{1}+1 / v_{r}}{1 / v_{2}+1 / v_{r}} l\right), l\right] .
$$

If the order of picking routes is sequenced as $L \rightarrow R \rightarrow D$, we have

$$
\begin{aligned}
& \frac{x^{k-1}}{v_{r}}+\frac{x^{k}}{v_{1}}=\frac{1-x^{k-1}}{v_{2}}+\frac{1-x^{k}}{v_{r}}, \\
& x^{k}=\left(1-x^{k-1}\right)\left(\frac{1 / v_{2}+1 / v_{r}}{1 / v_{1}+1 / v_{r}}\right) .
\end{aligned}
$$

Case 1. If

$$
\begin{gathered}
1-\frac{1 / v_{1}+1 / v_{r}}{1 / v_{2}+1 / v_{r}} l \geq 0, \\
x^{k-1} \in\left[1-\frac{1 / v_{1}+1 / v_{r}}{1 / v_{2}+1 / v_{r}} l, l\right]
\end{gathered}
$$

means

$$
\begin{gathered}
x^{k} \leq\left[1-\left(1-\frac{1 / v_{1}+1 / v_{r}}{1 / v_{2}+1 / v_{r}} l\right)\right]\left(\frac{1 / v_{2}+1 / v_{r}}{1 / v_{1}+1 / v_{r}}\right)=l, \\
x^{k} \geq(1-l)\left(\frac{1 / v_{2}+1 / v_{r}}{1 / v_{1}+1 / v_{r}}\right)>1-\frac{1 / v_{1}+1 / v_{r}}{1 / v_{2}+1 / v_{r}} l .
\end{gathered}
$$

The last inequality is because $s<l$. Thus,

$$
x^{k} \in\left[\max \left(0,1-\frac{1 / v_{1}+1 / v_{r}}{1 / v_{2}+1 / v_{r}} l\right), l\right] .
$$


Case 2. If

$$
\begin{gathered}
1-\frac{1 / v_{1}+1 / v_{r}}{1 / v_{2}+1 / v_{r}} l<0 \Longrightarrow \frac{1 / v_{2}+1 / v_{r}}{1 / v_{1}+1 / v_{r}}<l \\
x^{k-1} \in[0, l]
\end{gathered}
$$

means

$$
\begin{gathered}
x^{k} \leq\left(\frac{1 / v_{2}+1 / v_{r}}{1 / v_{1}+1 / v_{r}}\right)<l, \\
x^{k} \geq(1-l)\left(\frac{1 / v_{2}+1 / v_{r}}{1 / v_{1}+1 / v_{r}}\right)>0 .
\end{gathered}
$$

Thus, $x^{k} \in[0, l]$. Since $k$ is arbitrary, we conclude that if

$$
x^{0} \in\left[\max \left(0,1-\frac{1 / v_{1}+1 / v_{r}}{1 / v_{2}+1 / v_{r}} l\right), l\right] \text {, }
$$

then

$$
\begin{array}{r}
x^{k} \in\left[\max \left(0,1-\frac{1 / v_{1}+1 / v_{r}}{1 / v_{2}+1 / v_{r}} l\right), l\right], \\
\text { for } k=1,2,3, \ldots .
\end{array}
$$

Lemma 7 means that if

$$
x^{0} \in\left[\max \left(0,1-\frac{1 / v_{1}+1 / v_{r}}{1 / v_{2}+1 / v_{r}} l\right), l\right],
$$

the two trucks' hand-off locations $x^{k}$ are always on route $L$.

Lemma 8. If the tree-shaped picking system is sequenced as $L \rightarrow R \rightarrow D$ in Region 1 and

$$
x^{0} \in\left[\max \left(0,1-\frac{1 / v_{1}+1 / v_{r}}{1 / v_{2}+1 / v_{r}} l\right), l\right],
$$

then the two-truck bucket brigade picking system converges to the fixed point $x_{L}^{*}$.

Proof. According to Lemma 7, if

$$
x^{0} \in\left[\max \left(0,1-\frac{1 / v_{1}+1 / v_{r}}{1 / v_{2}+1 / v_{r}} l\right), l\right],
$$

then the hand-off location $x^{k}$ is always on route $L$. Let $x^{k}=$ $x_{L}^{*}+\delta^{k}$. Since

$$
\begin{gathered}
\frac{x^{k-1}}{v_{r}}+\frac{x^{k}}{v_{1}}=\frac{1-x^{k-1}}{v_{2}}+\frac{1-x^{k}}{v_{r}}, \\
\frac{x_{L}^{*}+\delta^{k-1}}{v_{r}}+\frac{x_{L}^{*}+\delta^{k}}{v_{1}}=\frac{1-\left(x_{L}^{*}+\delta^{k-1}\right)}{v_{2}}+\frac{1-\left(x_{L}^{*}+\delta^{k}\right)}{v_{r}}, \\
\delta^{k}=-\delta^{k-1}\left(\frac{1 / v_{2}+1 / v_{r}}{1 / v_{1}+1 / v_{r}}\right) \\
\delta^{k}=(-1)^{k}\left(\frac{1 / v_{2}+1 / v_{r}}{1 / v_{1}+1 / v_{r}}\right)^{k} \delta^{0} .
\end{gathered}
$$

Since $v_{1}<v_{2}$, as $k \rightarrow \infty,\left|\delta^{k}\right| \rightarrow 0$ and $x^{k} \rightarrow x_{L}^{*}$.
Lemma 9. If the tree-shaped picking system is sequenced as $R \rightarrow L \rightarrow D$ in Region 1, then there exists a unique fixed point on route $R$ and it is given by

$$
x_{R}^{*}=\frac{1 / v_{2}+1 / v_{r}}{1 / v_{1}+1 / v_{2}+2 / v_{r}}+l \text {. }
$$

Proof. If the order of picking routes is sequenced as $R \rightarrow$ $L \rightarrow D$, the fixed point $x_{R}^{*}$ can be found by solving

$$
\frac{x_{R}^{*}-l}{v_{1}}+\frac{x_{R}^{*}-l}{v_{r}}=\frac{1-\left(x_{R}^{*}-l\right)}{v_{2}}+\frac{1-\left(x_{R}^{*}-l\right)}{v_{r}} \text {, }
$$

$x_{R}^{*}=s+l, l<x_{R}^{*}<l+r$, so the fixed point is located on route $R$.

Lemma 10. If the tree-shaped picking system is sequenced as $R \rightarrow L \rightarrow D$ in Region 1 and

$$
x^{0} \in\left[l+\max \left(0,1-\frac{1 / v_{1}+1 / v_{r}}{1 / v_{2}+1 / v_{r}} r\right), l+r\right] \text {, }
$$

then

$$
\begin{array}{r}
x^{k} \in\left[l+\max \left(0,1-\frac{\frac{1}{v_{1}}+\frac{1}{v_{r}}}{\frac{1}{v_{2}}+\frac{1}{v_{r}}} r\right), l+r\right], \\
\text { for } k=1,2,3, \ldots
\end{array}
$$

Proof. The proof is the same as that in Lemma 7 except for the initial position

$$
x^{0} \in\left[l+\max \left(0,1-\frac{1 / v_{1}+1 / v_{r}}{1 / v_{2}+1 / v_{r}} r\right), l+r\right] \text {. }
$$

Lemma 10 shows that if

$$
x^{0} \in\left[l+\max \left(0,1-\frac{1 / v_{1}+1 / v_{r}}{1 / v_{2}+1 / v_{r}} r\right), l+r\right],
$$

the two trucks' hand-off locations $x^{k}$ are always on route $R$.

Lemma 11. If the tree-shaped picking system is sequenced as $R \rightarrow L \rightarrow D$ in Region 1 and

$$
x^{0} \in\left[l+\max \left(0,1-\frac{1 / v_{1}+1 / v_{r}}{1 / v_{2}+1 / v_{r}} r\right), l+r\right],
$$

then the two-truck bucket brigade picking system converges to the fixed point $x_{R}^{*}$.

Proof. The proof is the same as that in Lemma 8 except for the initial position

$$
x^{0} \in\left[l+\max \left(0,1-\frac{1 / v_{1}+1 / v_{r}}{1 / v_{2}+1 / v_{r}} r\right), l+r\right] \text {. }
$$




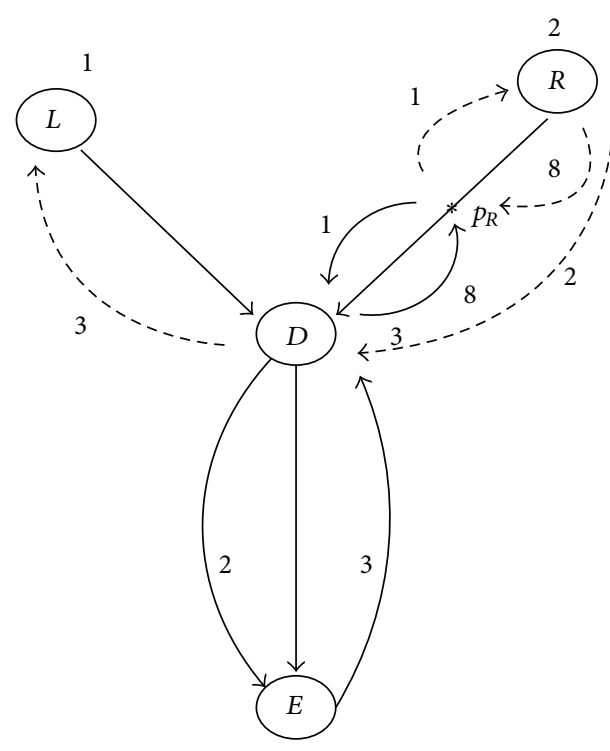

(a) Picking task is begun from node $L$

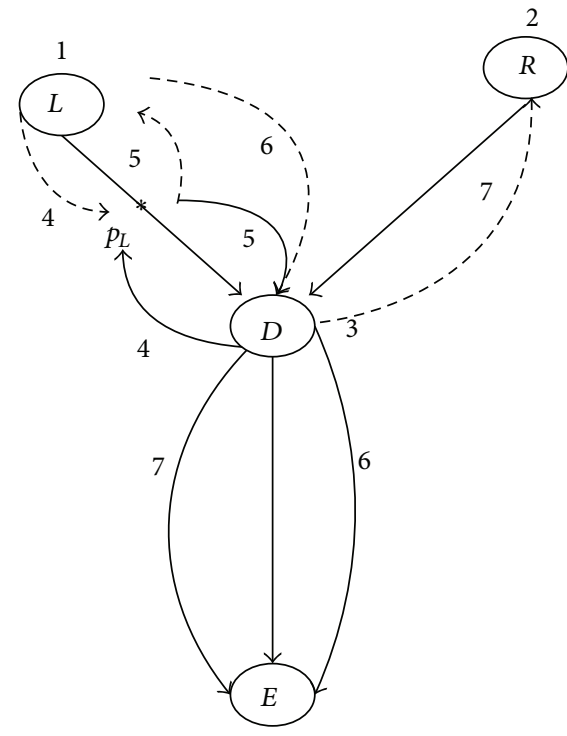

(b) Picking task is begun from node $R$

FIgURE 2: Period-2 orbit of the tree-shaped picking system.

3.2. Region $2(l<s<r)$. If $l<s<r$, the fixed point $x_{L}^{*}$ on route $L$ no longer exists; there is only one way to order the picking routes to achieve balance: $R \rightarrow L \rightarrow D$.

Lemma 12. If the tree-shaped picking system is sequenced as $R \rightarrow L \rightarrow D$ in Region 2, then the bucket brigade converges to a unique fixed point given by

$$
x_{R}^{*}=\frac{1 / v_{2}+1 / v_{r}}{1 / v_{1}+1 / v_{2}+2 / v_{r}}+l .
$$

Proof. The proof is the same as that in Lemmas 9 and 11.

3.3. Region $3(r<s<l+r)$. In regions, there is no ordering of picking routes in which the two-truck bucket brigade converges to a fixed point. But a period-2 orbit can be found if the order $L \rightarrow R \rightarrow D(R \rightarrow L \rightarrow D)$ is assigned to a period begun from node $L(R)$; then we can get a period-2 orbit on the tree-shaped picking system.

When the picking system runs on period- 2 orbit, the hand-offs occur periodically at two fixed positions $p_{L}$ and $p_{R}$ on route $L$ and route $R$, respectively. Figure 2 shows these hand-off positions. In Figure 2, the solid line represents the picking route of truck 1 , the dashed line represents the picking route of truck 2, and the number beside the line represents both trucks' walking sequence when they work as a bucket brigade. In Figure 2(a), the $k$ th picking task is begun from node $L$ and the picking system is sequenced as $L \rightarrow R \rightarrow$ $D$, truck 2 first completes the picking task on route $L$ and continues on route $R$ for $k$ period picking task. The $k$ th handoff occurs when truck 2 receives items collected in $k$ period from truck 1 at node $p_{R}$. After finishing $k$ period picking task, truck 1 goes back to node $R$ to begin its $k+1$ period picking, while truck 2 continues its $k$ period picking task. When truck 2 finishes the picking task on route $R$ for $k$ period, it goes to route $D$. Meanwhile, truck 1 is on $k+1$ period picking task on route $R$. Following the new order $R \rightarrow L \rightarrow D$ shown in Figure 2(b), truck 1 finishes the picking task on route $R$ and then goes to route $L$ to work on its $k+1$ period picking task before truck 2 completes $k$ period picking task.

The $(k+1)$ th hand-off occurs when truck 2 receives items collected in $k+1$ period from truck 1 at $p_{L}$. After the hand-off, truck 1 goes back to node $L$ to initiate $k+2$ period picking task, while truck 2 continues its $k+1$ period picking task. Following the order $L \rightarrow R \rightarrow D$ again, truck 1 will finish the picking task on route $L$ and go to route $R$ for $k+2$ period picking task before truck 2 finishes its $k+1$ period picking task. The $(k+2)$ th hand-off will be at $p_{R}$ again. So, the hand-offs of the two-truck picking system are repeatedly at two alternate locations $p_{L}$ and $p_{R}$.

Lemma 13. If the order of the tree-shaped picking system ( $L \rightarrow$ $R \rightarrow D$ or $R \rightarrow L \rightarrow D$ ) is assigned to a period picking task begun from node $L(R)$ in Region 3 , then there exists a unique period-2 orbit $p_{L}$ and $p_{R}$, where $p_{L}$ and $p_{R}$ are on route $L$ and route $R$, respectively, and they are given by

$$
\begin{gathered}
p_{R}^{*}=\frac{1 / v_{2}+1 / v_{r}}{1 / v_{1}+1 / v_{2}+2 / v_{r}}, \\
p_{L}^{*}=\frac{1 / v_{2}+1 / v_{r}}{1 / v_{1}+1 / v_{2}+2 / v_{r}}-r .
\end{gathered}
$$

Proof. If the hand-offs occur periodically at $p_{L}$ and $p_{R}$ as shown in Figure 2, $p_{L}$ and $p_{R}$ can be found by solving

$$
\begin{gathered}
\frac{p_{R}^{*}-l}{v_{r}}+\frac{r}{v_{1}}+\frac{l}{v_{r}}+\frac{p_{L}^{*}}{v_{1}}=\frac{1-p_{R}^{*}}{v_{2}}+\frac{1-\left(p_{L}^{*}+r\right)}{v_{r}}, \\
\frac{p_{R}^{*}}{v_{1}}+\frac{r}{v_{r}}+\frac{p_{L}^{*}}{v_{r}}=\frac{1-p_{R}^{*}}{v_{r}}+\frac{1-\left(p_{L}^{*}+r\right)}{v_{2}} .
\end{gathered}
$$


Furthermore, $p_{L}^{*}=s-r, p_{R}^{*}=s$, and $r<s<l+r$ means that $0<p_{L}^{*}<l$ and $r<p_{R}^{*}<l+r$. Thus, $p_{L}^{*}$ is on route $L$ and $p_{R}^{*}$ is on route $R$.

3.4. Region $4(s>l+r)$. When $s>l+r$ in Region 4 , there are two ways to order the picking routes: $L \rightarrow R \rightarrow D$ and $R \rightarrow L \rightarrow D$; both sequences of tree-shaped picking system can lead to a fixed point on route $D$.

Lemma 14. If the tree-shaped picking system is sequenced as $L \rightarrow R \rightarrow D$ or $R \rightarrow L \rightarrow D$ in Region 4, then there exists a unique fixed point on route $D$ and it is given by

$$
x_{D}^{*}=\frac{1 / v_{2}+1 / v_{r}}{1 / v_{1}+1 / v_{2}+2 / v_{r}} .
$$

Proof. If the tree-shaped picking system is sequenced as $L \rightarrow$ $R \rightarrow D$ or $R \rightarrow L \rightarrow D$, the fixed point $x_{D}^{*}$ can be found by solving

$$
\frac{x_{D}^{*}}{v_{1}}+\frac{x_{D}^{*}}{v_{r}}=\frac{1-x_{D}^{*}}{v_{2}}+\frac{1-x_{D}^{*}}{v_{r}},
$$

$x_{D}^{*}=s>l+r$, so the fixed point is located on route $D$.

Lemma 15. If the tree-shaped picking system is sequenced as $L \rightarrow R \rightarrow D$ or $R \rightarrow L \rightarrow D$ in Region 4 and

$$
x^{0} \in\left[l+r, 1-\left(\frac{1 / v_{1}+1 / v_{r}}{1 / v_{2}+1 / v_{r}}\right)(l+r)\right] \text {, }
$$

then

$$
x^{k} \in\left[l+r, 1-\left(\frac{1 / v_{1}+1 / v_{r}}{1 / v_{2}+1 / v_{r}}\right)(l+r)\right], \text { for } k=1,2,3, \ldots
$$

Proof. The proof is the same as that in Lemma 7, except for the initial position

$$
x^{0} \in\left[l+r, 1-\left(\frac{1 / v_{1}+1 / v_{r}}{1 / v_{2}+1 / v_{r}}\right)(l+r)\right] .
$$

Lemma 15 shows that if

$$
x^{0} \in\left[l+r, 1-\left(\frac{1 / v_{1}+1 / v_{r}}{1 / v_{2}+1 / v_{r}}\right)(l+r)\right],
$$

then the hand-off locations $x^{k}$ are always on route $D$.

Lemma 16. If the tree-shaped picking system is sequenced as $L \rightarrow R \rightarrow D$ or $R \rightarrow L \rightarrow D$ in Region 4 and

$$
x^{0} \in\left[l+r, 1-\left(\frac{1 / v_{1}+1 / v_{r}}{1 / v_{2}+1 / v_{r}}\right)(l+r)\right],
$$

then the two-truck bucket brigade picking system converges to the fixed point $x_{D}^{*}$.

Proof. The proof is the same as that in Lemma 8 except for the initial position

$$
x^{0} \in\left[l+r, 1-\left(\frac{1 / v_{1}+1 / v_{r}}{1 / v_{2}+1 / v_{r}}\right)(l+r)\right] .
$$

3.5. Summary. If the working and walk-back velocities of two trucks are given, the convergence analysis described in Sections 3.1 to 3.4 tells us when the two-truck bucket brigade tree-shaped picking system balances: Region 1 has two fixed points: one is on route $L$ and the other is on route $R$. Region 2 has two separate areas; the area on the left has a fixed point on route $R$ when $l \leq r$ and the right area has a fixed point on route $L$ when $l>r$. Region 3 has a period- 2 orbit and the hand-offs occur on route $L$ and route $R$ alternately and periodically; Region 4 only has one fixed point on route $D$. Because of the symmetry of the Y-shaped picking system, the case $l>r$ can also be found in Figure 1(b).

\section{Simulation}

4.1. Convergence Process Evolution. In all the simulations below, we set $v_{1}=1, v_{2}=1.5$, and $v_{3}=3$, respectively, and the convergence process of a two-truck bucket brigade tree-shaped system is observed in two ways: hand-off time evolution and hand-off position evolution. To show the handoff time evolution, we map hand-off location as a function of hand-off time, in which the horizontal axis and vertical axis represent the hand-off time and hand-off location at iteration $k+1$ of truck $i$ for $i=1,2$, respectively. And the Poincaré map is used to map the hand-off position evolution; the vertical axis in the map is the hand-off position at iteration $k+1$ of truck $i$ for $i=1,2$ and the horizontal axis is their hand-off position at iteration $k$; the red dashed line in the Poincaré map shows the diagonal, and the solid line with arrows traces the hand-off position evolution.

4.1.1. Region $1(s<l<r, l=0.45)$. In Region 1 , if the picking routes are sequenced as $L \rightarrow R \rightarrow D$, simulation results of the convergence process are shown in Figure 3.

Figure 3(a) shows that the hand-off locations $x^{k}$ converge quickly to a single point $x_{L}^{*}=0.4286$ on the left subpicking route $L \rightarrow D$. The hand-off position evolution in the Poincaré map is shown in Figure 3(b) when the initial iterate $x^{1}=0.9$; the intersection of the diagonal with the Poincare map at a point $x_{L}^{*}=0.4286$ indicates a fixed point on subpicking route $L \rightarrow D$, which implies that truck 1 always works from point 0 to $x_{L}^{*}$ and truck 2 from $x_{L}^{*}$ to 1 . Hence, trucks always perform the same amount of picking task in each picking period.

In Region 1 , if the picking routes are sequenced as $R \rightarrow$ $L \rightarrow D$, simulation results of the convergence process are shown in Figure 4.

Figure 4(a) shows that the hand-off locations $x^{k}$ converge quickly to a single point $x_{R}^{*}=0.8786$ on the right subpicking route $R \rightarrow D$. The hand-off position evolution in the Poincaré map is shown in Figure 4(b) when the initial iterate $x^{1}=0.8$; the intersection of the diagonal with the Poincare map at a point $x_{R}^{*}=0.8786$ indicates a fixed point on subpicking route $R \rightarrow D$, which implies that truck 1 always works from point 0 to $x_{R}^{*}$ and truck 2 from $x_{R}^{*}$ to 1 . Hence, trucks always perform the same amount of tasks in each picking period. 


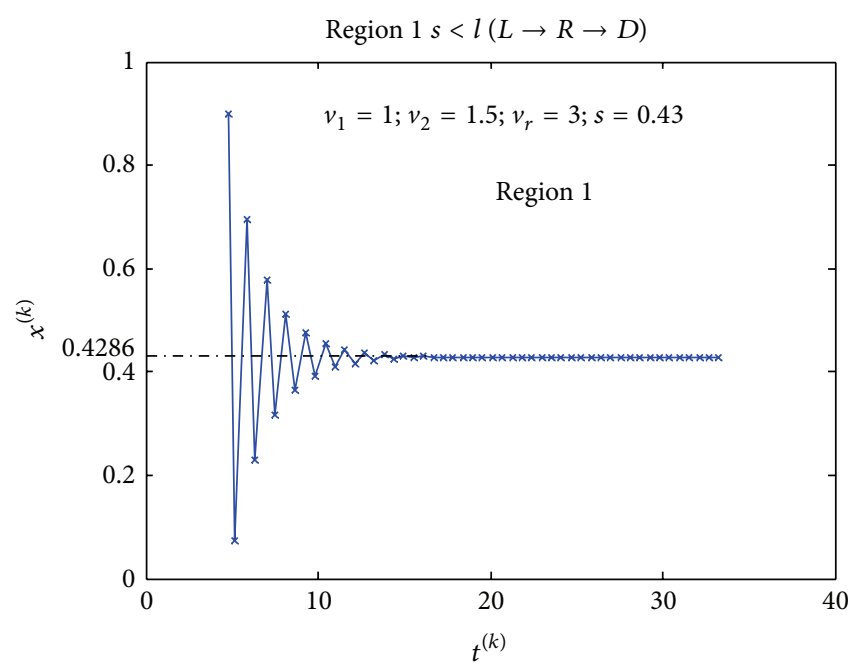

(a) Hand-off time evolution

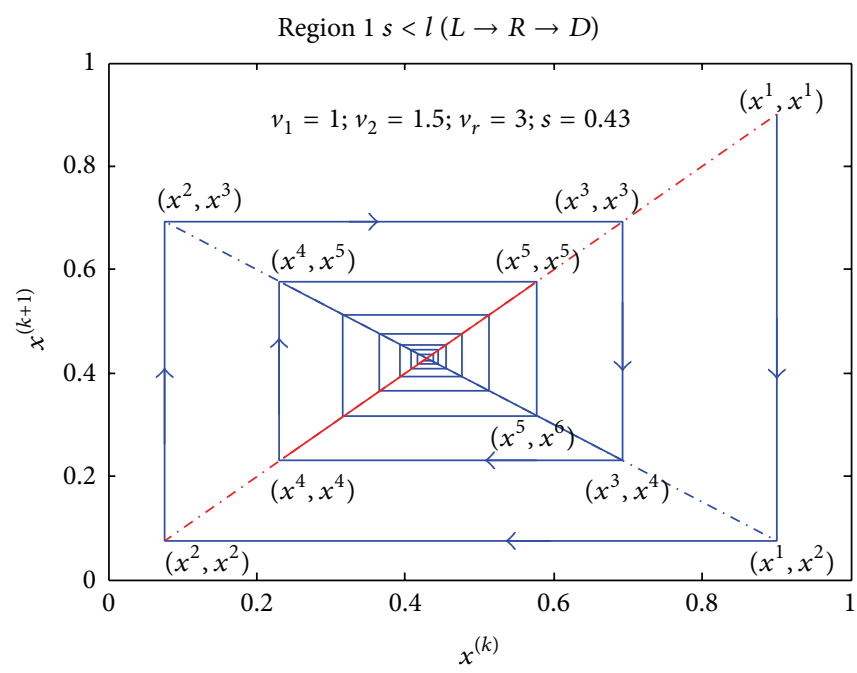

(b) Hand-off position evolution

FIgURE 3: Convergence process in Region 1 when the picking routes are sequenced as $L \rightarrow R \rightarrow D$.

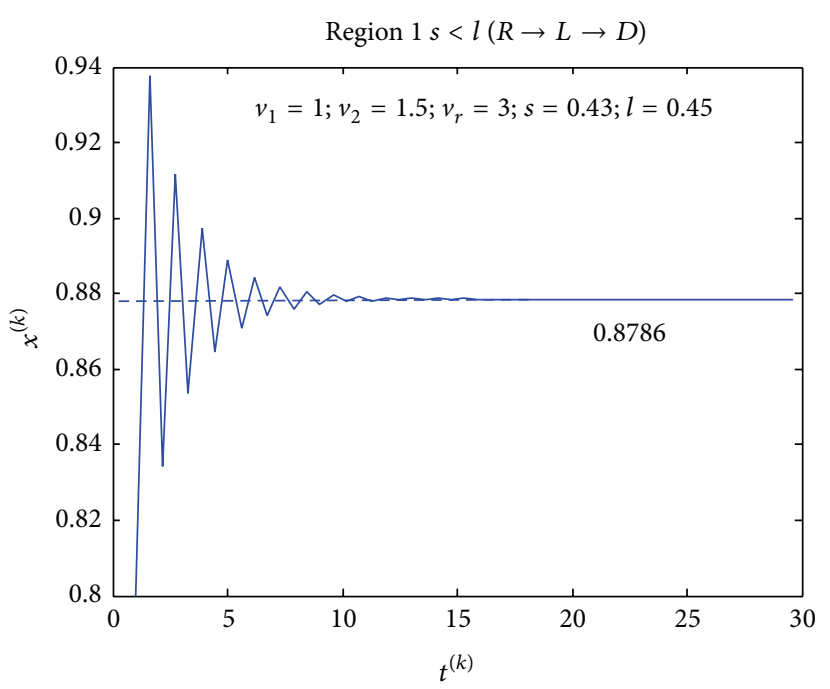

(a) Hand-off time evolution

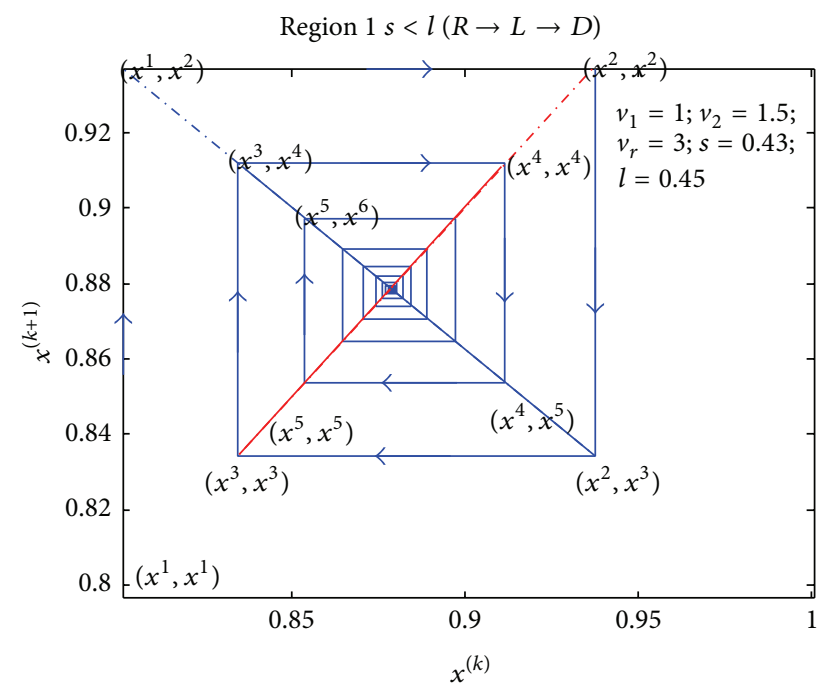

(b) Hand-off position evolution

FIgURE 4: Convergence process in Region 1 when the picking routes are sequenced as $R \rightarrow L \rightarrow D$.

4.1.2. Region $2(l<s<r, l=0.4)$. In Region 2, the picking routes can only be sequenced as $R \rightarrow L \rightarrow D$. Simulation results of the convergence process in Region 2 when the picking routes are sequenced as $R \rightarrow L \rightarrow D$ are the same as those in Figure 4.

4.1.3. Region $3(r<s<l+r, l=0.2 ; r=0.3)$. In Region 3, if the picking routes are sequenced as $(L \rightarrow R \rightarrow D$ or $R \rightarrow$ $L \rightarrow D)$ and assigned to a period picking task begun from node $L(R)$ accordingly, simulation results of the convergence process are shown in Figure 5.

As shown in Figure 5(a), the hand-off locations $x^{k}$ eventually alternately converge to a period-2 orbit: $P_{L}^{*}=$ 0.1286 and $P_{R}^{*}=0.4286$. The Poincaré map in Figure 5(b) converges to period- 2 orbit when the initial iterate $x^{1}=$ 0.3 . Once the two-truck bucket brigade tree-shaped system converges to the period- 2 orbit, truck 2 takes over the picking task from truck 1 at points $P_{L}^{*}=0.1286$ and $P_{R}^{*}=0.4286$ alternately.

4.1.4. Region $4(s>l+r)$. In Region 4 , there is no difference between the convergence process whether the picking routes are sequenced as $L \rightarrow R \rightarrow D$ or $R \rightarrow L \rightarrow D$. Simulation results of the convergence process are shown in Figure 6.

As shown in Figure 6(a), the hand-off locations $x^{k}$ converge quickly to a single point $x_{D}^{*}=0.4286$ on subpicking route $D \rightarrow E$. The hand-off position evolution in the Poincaré map is shown in Figure 6(b) when the initial iterate 


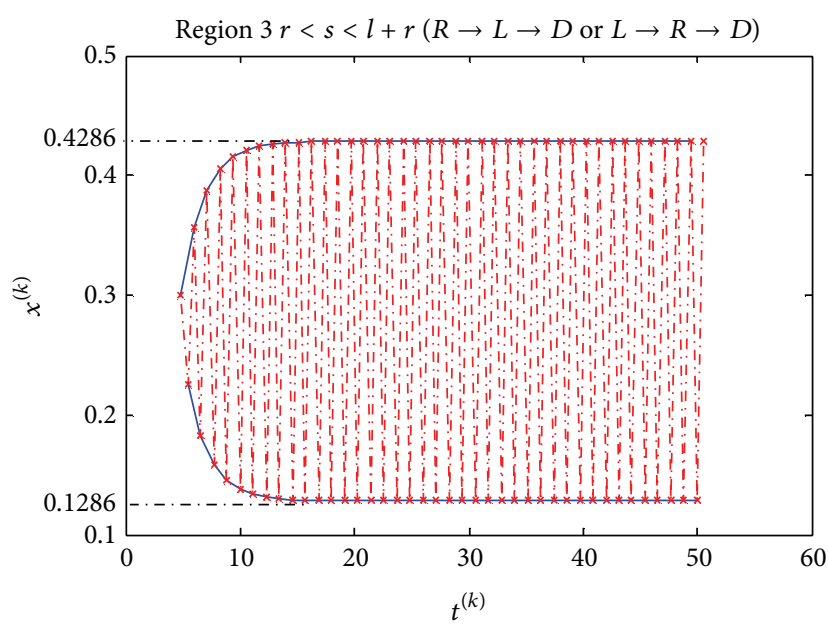

(a) Hand-off time evolution

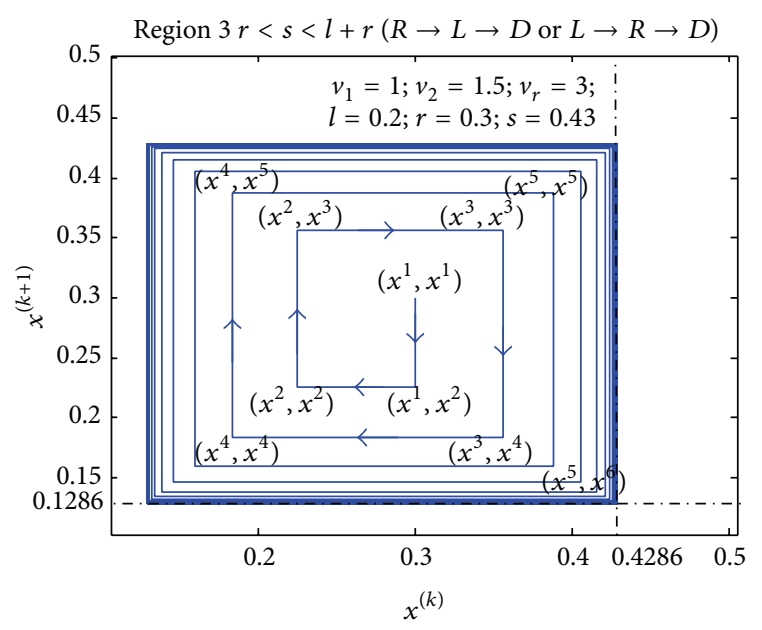

(b) Hand-off position evolution

FIGURE 5: Convergence process in Region 3.

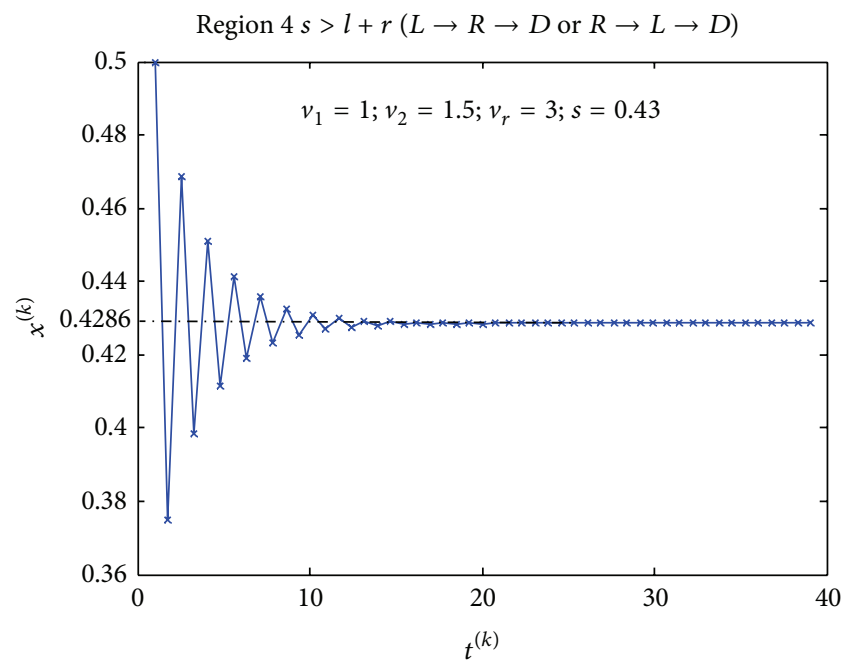

(a) Hand-off time evolution

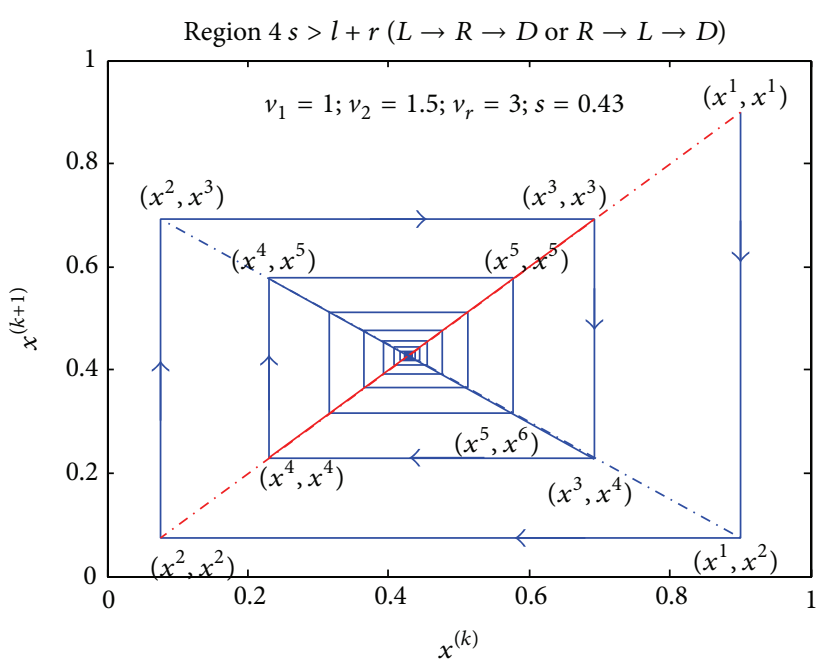

(b) Hand-off position evolution

FIGURE 6: Convergence process in Region 4.

$x^{1}=0.9$. The intersection of the diagonal with the Poincare map at a point $x_{D}^{*}=0.4286$ indicates a fixed point on subpicking route $D \rightarrow E$. The fixed point for the two-truck bucket brigade tree-shaped picking system implies that truck 1 always works from point 0 to $x_{D}^{*}$ and truck 2 from $x_{D}^{*}$ to 1. Hence, trucks always perform the same amount of tasks in each picking period.

4.2. Factors Affecting Convergence. According to the lemmas in Section 3, the range of $x^{k}$ is determined when initial conditions $\left(v_{1}, v_{2}, v_{r}, l, r\right.$, and $\left.d\right)$ are given. The question that naturally comes to mind is whether the system's initial condition affects the behavior of the two-truck bucket brigade tree-shaped picking system, so, in this section, we, therefore, want to know how initial conditions affect the stable behavior of the bucket brigade. We focus on two factors of initial conditions: initial location $x^{1}$ and the working velocity difference of two trucks. In order to observe the effect of initial location on the system's behavior, we simulate the system's behavior under different initial locations $x^{1}$ and different $v_{1}$ and $v_{2}$, respectively. Simulation results are shown in Figures 7 and 8, respectively.

Figure 7 tells us that the two-truck bucket brigade treeshaped picking system converges quickly to a single point no matter where the initial node $x^{1}$ is located. This implies that the initial location of the trucks is irrelevant. More precisely, the two-truck bucket brigade picking system always converges to a fixed point no matter which node the trucks start to work at.

Figure 8 tells us that although the two-truck bucket brigade tree-shaped picking system can converge to a single point when the conditions in each region hold, the working 

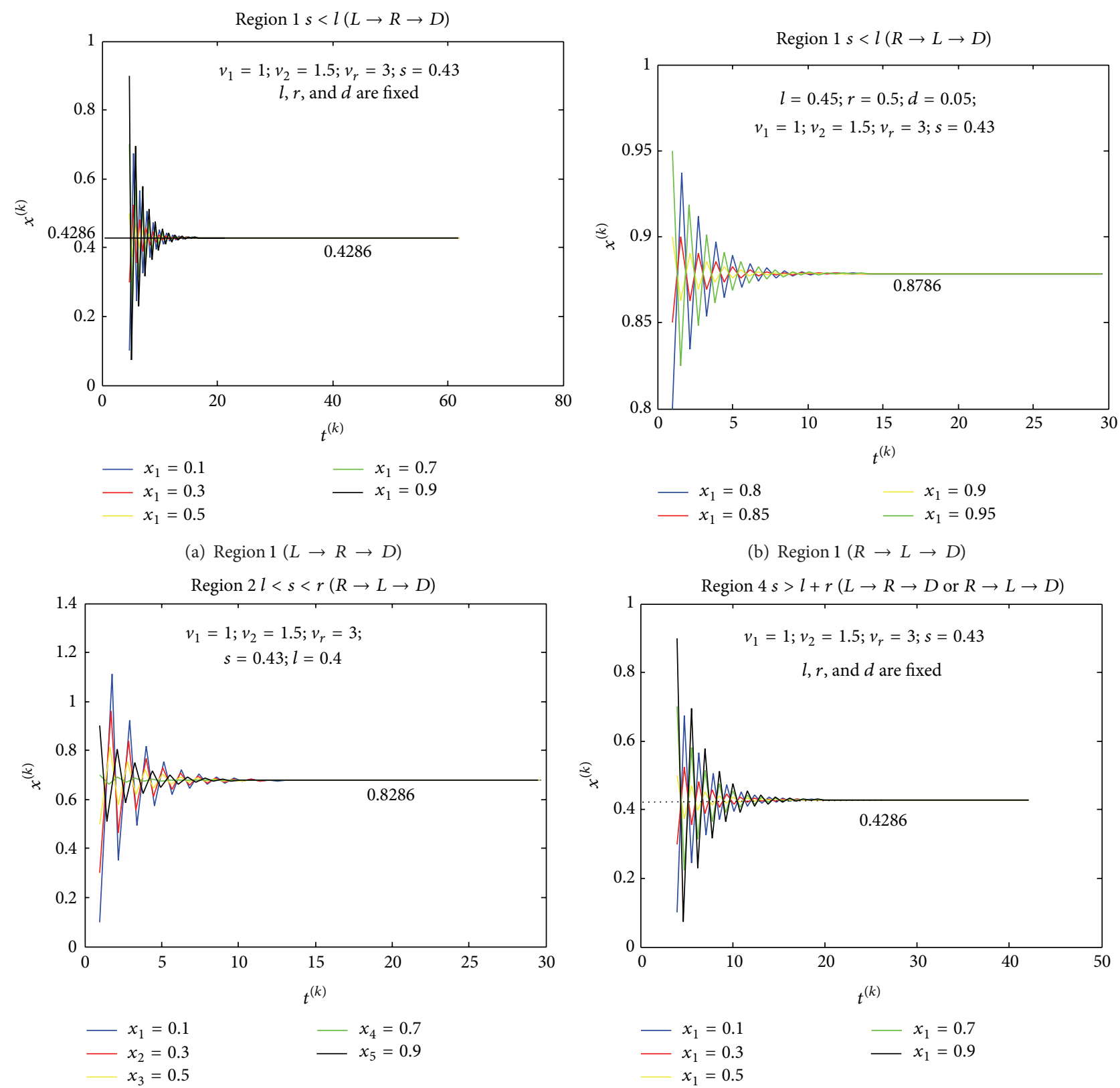

(b) Region $1(R \rightarrow L \rightarrow D)$

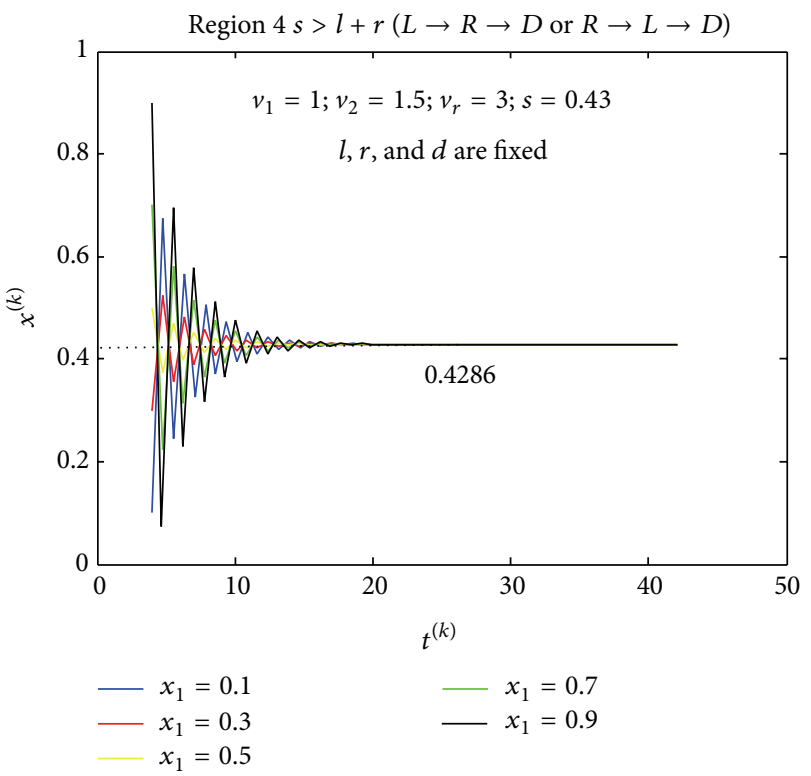

(c) Region $2(R \rightarrow L \rightarrow D)$

(d) Region $4(L \rightarrow R \rightarrow D / R \rightarrow L \rightarrow D)$

FIGURE 7: Effect of initial location on system's stable behavior.

velocity difference plays a major role in the speed of the system's convergence process. The bigger the difference between the two trucks' working velocities is, the faster the two-truck bucket brigade tree-shaped picking system converges.

4.3. Managerial Implication. Lemmas 6 to 16 in Section 3 and simulation in Section 4 tell us that if two trucks follow the bucket brigade rule in a tree-shaped picking system, the treeshaped picking system will always be self-organizing, which means that significant managerial intervention regarding the initial location is not necessary for effective operation of the two-truck bucket brigade. But simulations in Section 4.2 tell us clearly that a picking system with large differences in trucks' working velocities can easily reach self-balance status, which means that some managerial intervention is necessary regarding the difference in the trucks' velocity in order to enhance the self-organization of the bucket brigade picking system.

\section{Conclusions and Further Work}

The main contribution of this paper is to show how the idea of the bucket brigade can be applied to tree-shaped picking systems. We achieve this by conceptually converting the work 

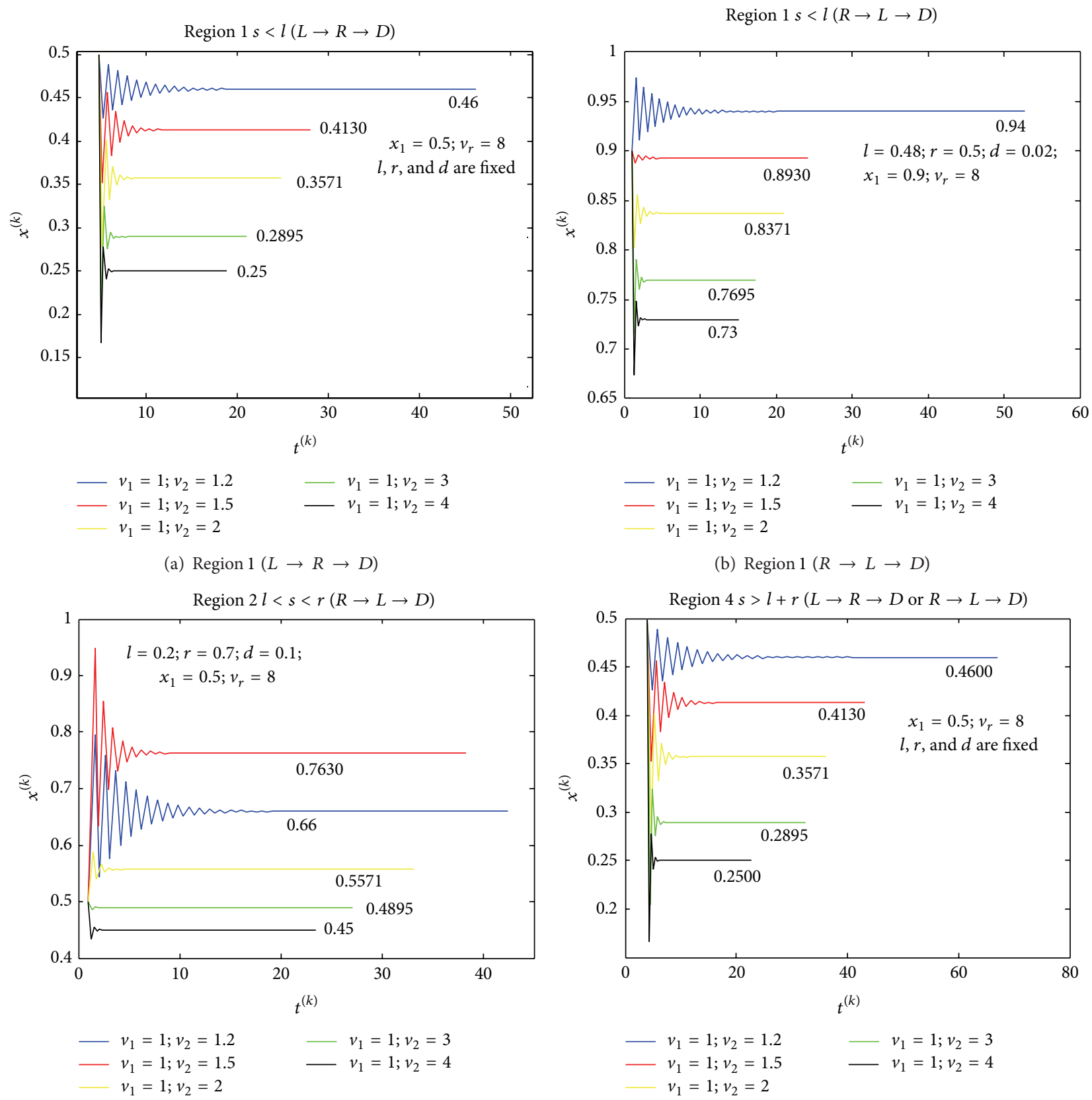

(c) Region $2(R \rightarrow L \rightarrow D)$

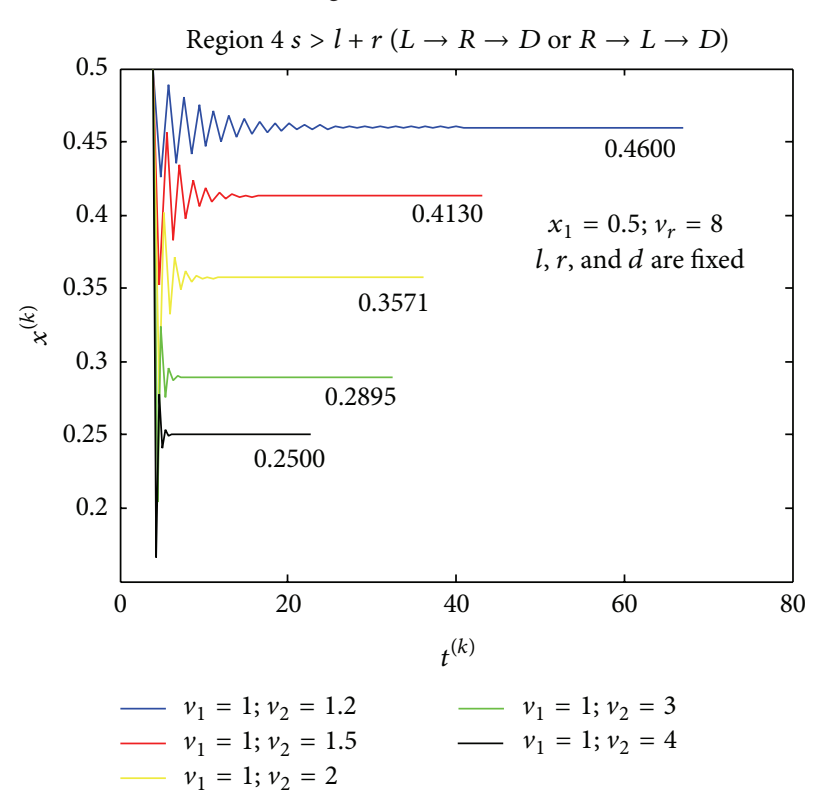

(d) Region $4(L \rightarrow R \rightarrow D / R \rightarrow L \rightarrow D)$

FIGURE 8: Effect of working velocity difference on system's stable behavior.

content of a tree-shaped picking system to a linear sequence of work to which the adapted bucket brigade protocol can be applied. We find that if two trucks follow the bucket brigade rule in a tree-shaped picking system, the tree-shaped picking system will always be self-organizing; that is, trucks hand over picking items at a fixed point or at two fixed locations that they visit periodically. Our work complements that of Bartholdi III et al. [13] by introducing significant walk-back time into a tree-shaped bucket brigade system and, moreover, our analysis provides a way to model and understand bucket brigade picking systems.
In this paper, the picking routes are assumed to be Yshaped. In actual situations, however, the picking route is more complex than a Y-shaped tree; it can even be a network. Thus, picking networks and $n$-trucks $(n>2)$ bucket brigade picking systems could be considered in future research.

\section{Conflict of Interests}

The authors declare that there is no conflict of interests regarding the publication of this paper. 


\section{Acknowledgments}

The work is supported by the National Natural Science Foundation of China under Grant no. 711712000, the Natural Science Foundation of Jiangxi Province, China, under Grant no. 20114BAB201029, the Scientific Foundation of Educational Department of Jiangxi Province, China, under Grant no. GJJ12283, and the Foundation of East China Jiaotong University under Grant nos. 11JD08, 13JD04.

\section{References}

[1] J. J. Bartholdi III and D. D. Eisenstein, "A production line that balances itself," Operations Research, vol. 44, no. 1, pp. 21-34, 1996.

[2] B. J. Schroer, J. Wang, and M. C. Ziemke, "A look at TSS through simulation,” Bobbin, vol. 7, pp. 114-119, 1991.

[3] J. J. Bartholdi III, D. D. Eisenstein, and R. D. Foley, "Performance of bucket brigades when work is stochastic," Operations Research, vol. 49, no. 5, pp. 710-719, 2001.

[4] L. F. Muñoz and J. R. Villalobos, "Work allocation strategies for serial assembly lines under high labour turnover," International Journal of Production Research, vol. 40, no. 8, pp. 1835-1852, 2002.

[5] J. J. Bartholdi III, D. D. Eisenstein, and Y. F. Lim, Chaos and Convergence in Bucket Brigades with Finite Walk-Back Velocities, Georgia Institute of Technology, Atlanta, Ga, USA, 2004.

[6] D. Armbruster and E. S. Gel, "Bucket brigades revisited: are they always effective?" European Journal of Operational Research, vol. 172, no. 1, pp. 213-229, 2006.

[7] D. Armbruster, E. S. Gel, and J. Murakami, "Bucket brigades with worker learning," European Journal of Operational Research, vol. 176, no. 1, pp. 264-274, 2007.

[8] J. J. Bartholdi III and D. D. Eisenstein, "Using bucket brigades to migrate from craft manufacturing to assembly lines," Manufacturing and Service Operations Management, vol. 7, no. 2, pp. 121-129, 2005.

[9] S. T. Hutchinson, J. R. Villalobos, and M. G. Beruvides, "Effects of high labour turnover in a serial assembly environment," International Journal of Production Research, vol. 35, no. 11, pp. 3201-3223, 1997.

[10] J. J. Bartholdi III and D. D. Eisenstein, Bucket Brigades: A Self-Balancing Order-Picking System for a Warehouse, Georgia Institute of Technology, Atlanta, Ga, USA, 1996.

[11] Y. F. Lim, "Cellular bucket brigades," Operations Research, vol. 59, no. 6, pp. 1539-1545, 2011.

[12] Y. F. Lim, Some generalizations of bucket brigade assembly lines [Ph.D. thesis], Georgia Institute of Technology, School of Industrial and Systems Engineering, 2005.

[13] J. J. Bartholdi III, D. D. Eisenstein, and Y. F. Lim, "Bucket brigades on in-tree assembly networks," European Journal of Operational Research, vol. 168, no. 3, pp. 870-879, 2006. 


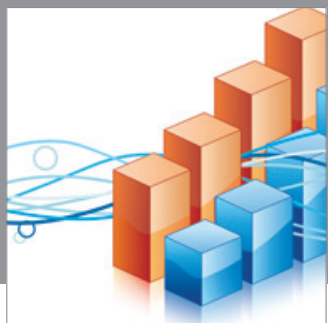

Advances in

Operations Research

mansans

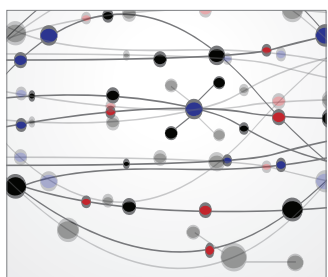

The Scientific World Journal
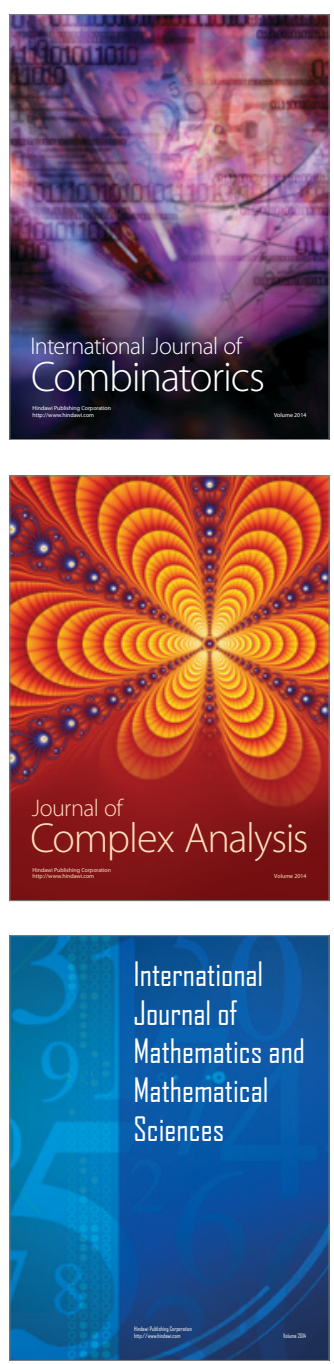
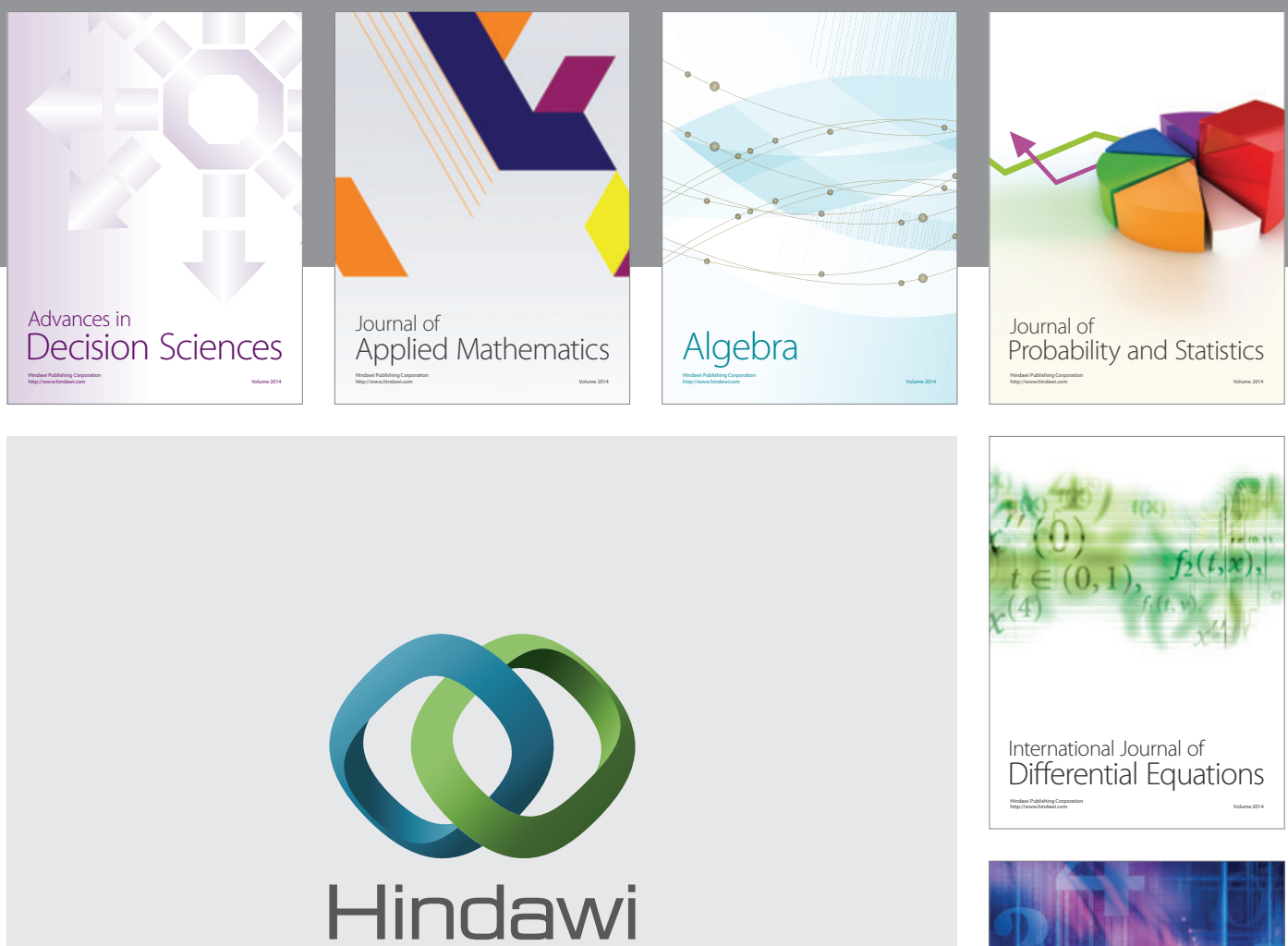

Submit your manuscripts at http://www.hindawi.com
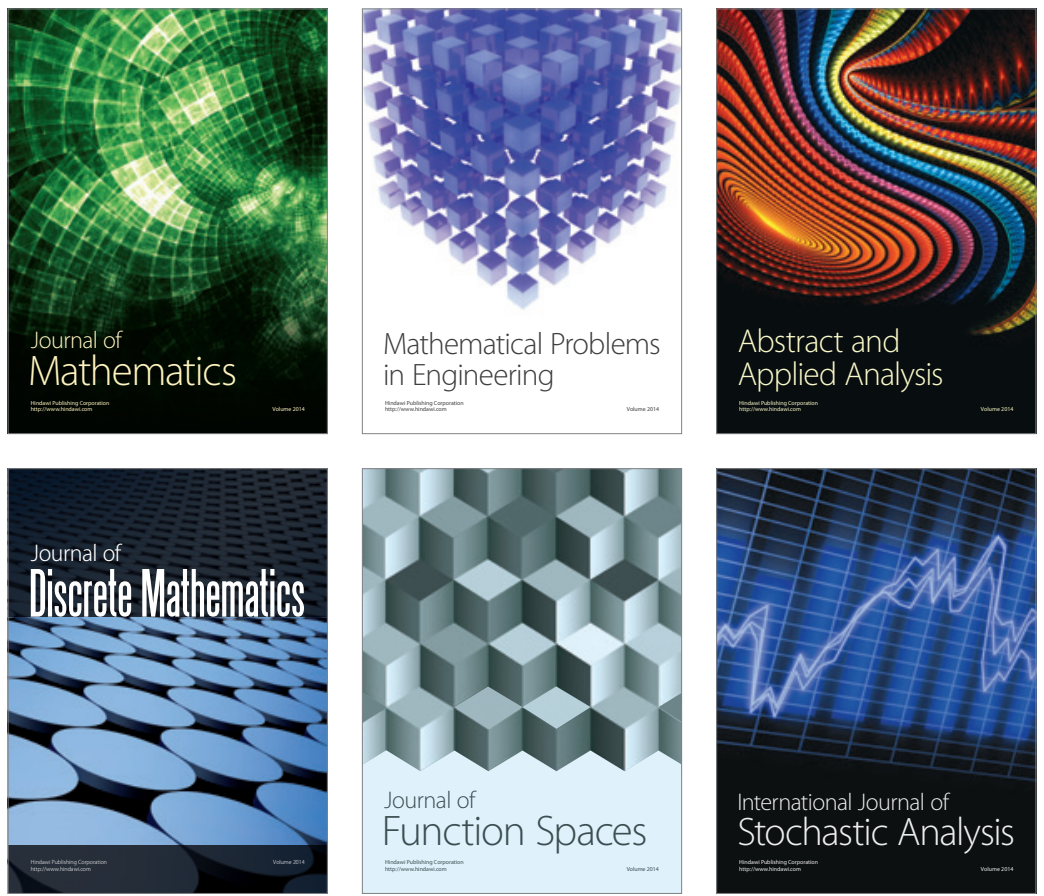

Journal of

Function Spaces

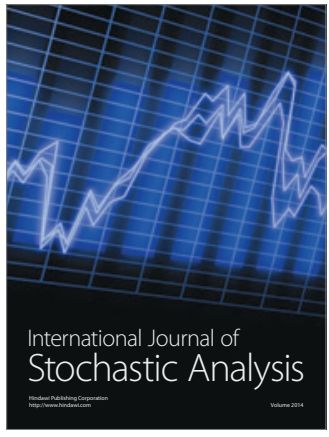

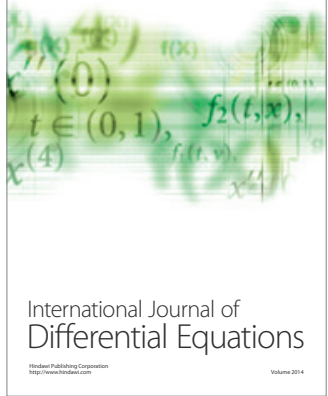
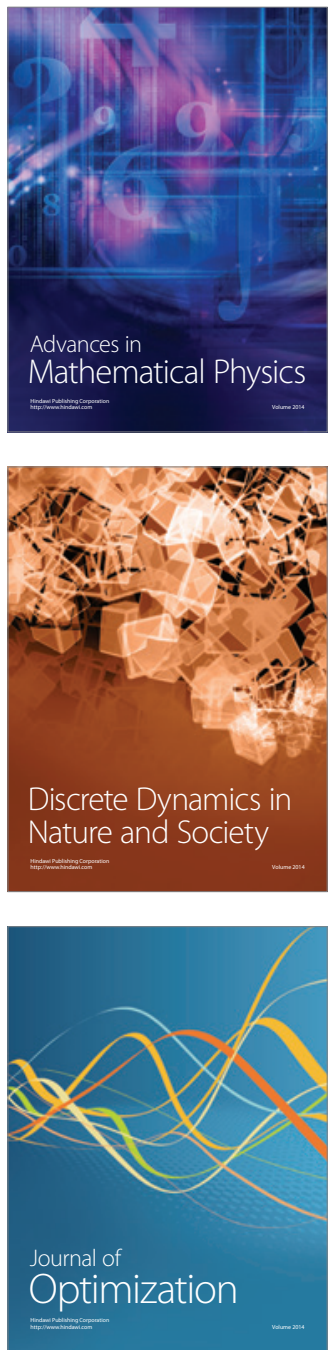